\section{Estimation of Ammonia Volatilized from Soils}

Axthough it has been recognized that a part of the nitrogen in the soil system may be lost in the form of ammonia, the extent of such loss--especially under field conditions - has not yet been satisfactorily determined, largely owing to want of suitable technique. Estimates based on difference in total nitrogen are not sufficiently accurate. Aeration methods are tedious and time consuming : they also disturb the biological conditions in the medium. Extraction and distillation methods are unsuitable, as the estimates thus obtained may not be entirely due to free ammonia. The following method was therefore developed, and was found to work quite satisfactorily.

The procedure consists in pasting a piece of filter paper (previously acid treated, for example, Whatman No. 30 or 41 ) moistened with standard acid on the inside of a glass or enamelled dish which just covers the beaker or other vessel containing the soil. The ammonia evolved from the medium is absorbed by the acid. At convenient intervals the dish can be removed and the unused acid in the paper estimated by back titration against standard alkali with methyl red as indicator and boiling to drive off carbon dioxide.

The above procedure is very simple and rapid. By suitably altering the strength of acid, varying quantities of ammonia can be estimated. The method can be applied to time studies, the paper being changed periodically. The temperature can also be varied according to requirements. The studies can be carried out under both laboratory and field conditions.

Applying the above method, it has been found that practically all soils treated with ammoniacal fertilizers-especially under tropical conditions-lose ammonia by volatilization. In some cases, all the added nitrogen is lost in the course of ten days. Soil treated with organic manures with narrow $\mathrm{C}-\mathrm{N}$ ratios also lose large quantities of nitrogen as ammonia.

The mechanism of the related processes has been studied and will be reported elsewhere.

Indian Institute of Science,

$$
\text { V. Subrahmanyan. }
$$

Bangalore.

April 7.

\section{The So-called 'Transition Temperature' of Metallic Films}

THE relation of the electric resistance to temperature of metallic films of zinc, cadmium, magnesium and antimony, condensed from vapours on a cold surface of glass, was investigated employing an apparatus similar to that described in a previous paper ${ }^{1}$. A double-walled copper cylinder was inserted in the inner vessel in order to cool pentane contained in the latter indirectly with liquid nitrogen, and a stirrer was used to ensure uniformity of temperature distribution. The bath temperature was read with a copper-constantan thermo-junction and a millivoltmeter, and the thicknesses of the metallic films were estimated by weighing with a microbalance having an accuracy of $1 \times 10^{-5} \mathrm{gm}$. The metallic specimens were from Kahlbaum.

Zinc, cadmium and magnesium were condensed on glass at $-150^{\circ} \mathrm{C}$. and antimony at $-75^{\circ} \mathrm{C}$., and the heating rates were kept between $1 \cdot 0^{\circ} \sim 1 \cdot 2^{\circ} \mathrm{C}$. per minute. An example of the changes of resistance for various thicknesses of films is shown in Fig. 1. The so-called 'transition temperature' 2, which is the crystallizing temperature, is indicated by arrows, and becomes less distinct when the thicknesses of the

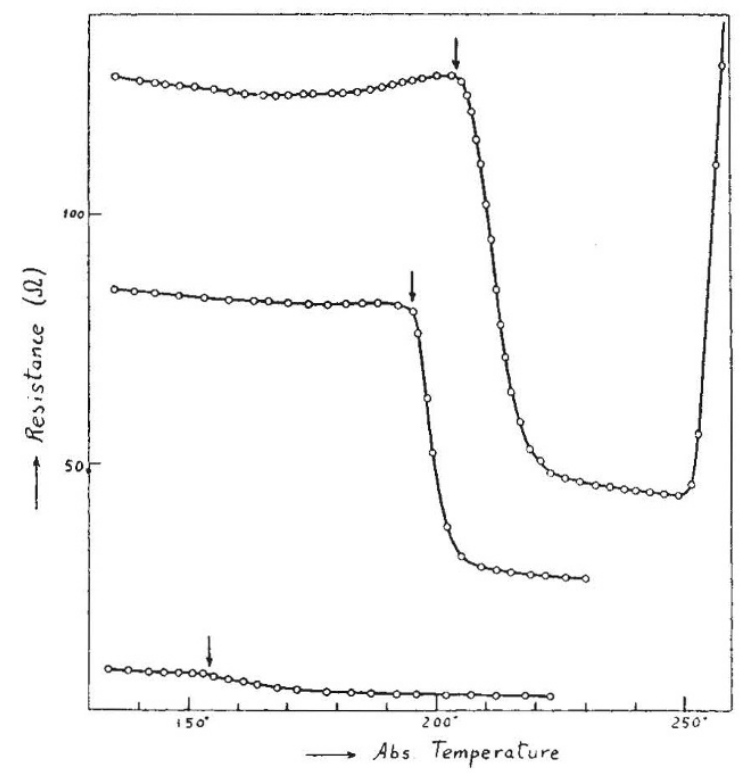

Fig. 1.

Reststance of cadmitm films.

films reach several hundreds millimicrons. Abrupt increases of resistance in zinc, cadmium and mag. nesium are caused from the crack-formation in the films.

If the films are not too thin, linear relations exist between the thicknesses and the logarithm of the transition temperatures. The gradients of these straight lines are exactly the same for zinc and cadmium, and almost the same for antimony and magnesium. When this straight line relation is extrapolated to zero thickness, the ratio of transition temperature $(T u)$ to melting point $(T s)$ of the respective metals ranges from $0 \cdot 36$ to $0 \cdot 4 \mathrm{l}$, which coincides with that for the bulk metals ${ }^{3}$ as shown in the following table:

\begin{tabular}{|l|l|c|c|c|}
\hline & Zinc & Cadmium & Magnesium & Antimony \\
\hline loge $t u$ & $5 \cdot 625$ & $5 \cdot 485$ & $5 \cdot 8$ & $5 \cdot 82$ \\
$t_{u}$ & $277^{\circ}$ & $241^{\circ}$ & $330^{\circ}$ & $337^{\circ}$ \\
$t_{s}$ & $692^{\circ}$ & $591^{\circ}$ & $906^{\circ}$ & $903^{\circ}$ \\
$t_{u} / T_{s}$ & $0 \cdot 40$ & $0 \cdot 41$ & $0 \cdot 36$ & $0 \cdot 37$ \\
\hline
\end{tabular}

The present investigation is being extended to other metals.

Research Institute for Iron,

TADaO FukUror.

Steel and Other Metals,

Sendai.

March 1 .

${ }^{1}$ T. Fukuroi, Sci. Rep. Tôhoku Imp. Cniv., Prof. Honda Anniversary Volume, 80 (1936).

${ }^{2}$ Kramer, J., Ann. Phys., (5), 19, 37 (1934) ; Naturwiss., 20, 792, etc. (1932).

${ }^{3}$ Tammann, G., $Z$. anorg. Chem., 157, 325 (1926) or Jefferies and Archer, "The Science of Metals"' (1924), p. 86. The ratios of the $T R / T^{s}=0.32 \sim 0 \cdot 43$ for ordinary metals. 